Applied Mathematical Sciences, Vol. 14, 2020, no. 9, 409 - 416

HIKARI Ltd, www.m-hikari.com

https://doi.org/10.12988/ams.2020.914211

\title{
Infinite-Horizon Linear-Quadratic Age-Structured Optimal Control Problem
}

\author{
Alessandra Buratto, Rudy Cesaretto, and Luca Grosset* \\ Dipartimento di Matematica "Tullio Levi-Civita" \\ Università degli Studi di Padova, Italy
}

This article is distributed under the Creative Commons by-nc-nd Attribution License. Copyright (c) 2020 Hikari Ltd.

\begin{abstract}
In this paper we show how to apply the sufficient conditions to a simple infinite-horizon linear-quadratic age-structured optimal control problem. Our approach is based on the current-value Hamiltonian and it leads us to study a linear system of PDEs. Using the standard theory of Riccati equation we can simplify this problem decoupling the system of PDEs. When the model is autonomous, this theory allows us to find the optimal equilibrium by a Riccati ODE. This approach can be the starting point to face linear-quadratic age-structured differential games.
\end{abstract}

Mathematics Subject Classification: 49N10, 49K20, 91B55

Keywords: Age-structured dynamics, Linear-quadratic optimal control

\section{Introduction}

Age is one of the most natural and important parameters in structuring dynamics. Many internal variables at the individual level are intimately dependent on age. For a long time, the interest in age structure was restricted to demography, but nowadays it plays a fundamental role in other fields such as Ecology, Epidemiology, Biology, and even Economics. Age-structured models have been used to formalize and solve optimal control problems in different fields: e.g.

\footnotetext{
${ }^{*}$ Corresponding author
} 
drug initiation [1], dynamic advertising [8], vintage capital [6], immigration policies [14], and population dynamics [2], [10] .

Necessary conditions have been introduced and applied to analyze different kinds of models, see e.g. [5] and [15]. Mangasarian-type sufficient conditions for age-structured optimal control problems are presented by Krastev in [11], and Arrow-type sufficient conditions are described in [12]. Moreover, in [3] sufficient conditions using the current-value Hamiltonian are designed to solve a typical age-structured optimal control problem with a discount factor in the objective functional.

The class of linear-quadratic (LQ) optimal control problems, i.e. the optimal control of linear systems with a quadratic cost function, can model many problems in applications, and many nonlinear control problems can be reasonably approximated by LQ problems. Moreover, solutions of LQ problems exhibit elegant properties due to their simple and nice structures. This topic has been extensively studied in the literature, characterizing the optimal solutions in different contexts: continuous, discrete, finite and infinite-horizon, deterministic and stochastic, see e.g. [7] and [4].

In this paper, we tackle a linear-quadratic age-structured optimal control problem, over an infinite-horizon with a discount factor in the objective functional. Initially, we apply the sufficient conditions based on the current-value Hamiltonian for an age-dependent optimal control problem as described in [3]. In order to find an optimal control we have to solve a linear system of PDEs. Using the standard theory of Riccati equation we show that the obtained linear system of PDEs can be decoupled. This approach is useful if we want to find an equilibrium point for an autonomous model because the solution is reduced to the analysis of a Riccati ODE.

The paper is organized as follows: In Section 2 we introduce the linearquadratic age-structured optimal control problem we are dealing with. In Section 3 we characterize the optimal solution throughout the sufficient conditions based on the current value Hamiltonian. Finally, Section 4 concludes the paper with some suggestions about the further research on this field.

\section{LQ age-structured optimal control problem}

Let us consider the following quadratic objective functional to be maximized

$$
J(u)=\int_{0}^{+\infty} \int_{0}^{\omega} e^{-\rho t}\left[\frac{\varepsilon(a)}{2} y^{2}(t, a)+\frac{\zeta(a)}{2} u^{2}(t, a)\right] d a d t .
$$

The LQ age-structured dynamics of the problem is quite simple: The state variable $y(t, a)$ evolves according to the following linear autonomous partial differential equation

$$
\partial_{t} y(t, a)+\partial_{a} y(t, a)=\alpha(a) y(t, a)+\beta(a) u(t, a) .
$$


The initial condition for all $a \in[0, \omega]$ is represented by the boundary condition

$$
y(0, a)=\gamma(a),
$$

while the boundary condition at zero age for all $t \in(0,+\infty)$ is described by

$$
y(t, 0)=\delta(t)
$$

The motion equation is linear both in the state and in the control, hence, for any control $u(t, a) \in C^{0}([0,+\infty) \times[0, \omega] ; \mathbb{R})$ there exists a unique state function $y(t, a)$ defined in $[0,+\infty) \times[0, \omega]$ that satisfies $(2),(3)$, and $(4)$.

For the reader's convenience we sketch the procedure that allows to find the state function associated to a given control. The inflow characteristics associated with the PDE (2) are the lines $a=t+a_{0}$ for any $a_{0} \in \mathbb{R}$; hence, using the information on the segment $\{0\} \times[0, \omega]$ and on the semi-axis $(0,+\infty) \times\{0\}$, we can find a solution of (2) by the method of characteristics [13, Ch.4, p.165]. Let $P=(t, a)$ be a point in the domain of the state function and let $u(t, a) \in$ $C^{0}([0,+\infty) \times[0, \omega] ; \mathbb{R})$ be a feasible control.

If $t \leq a$ then the characteristic line passing through point $\mathrm{P}$ has equation: $\sigma=(s-t)+a$. When $s=0$, this characteristic line assumes the following form: $\sigma=-t+a \geq 0$. Hence, if we define the function $x(s)=y(s, s-t+a)$, then its derivative w.r.t. $s$ is

$$
\begin{aligned}
\dot{x}(s) & =y_{t}(s, s-t+a)+y_{a}(s, s-t+a) \\
& =\alpha(s-t+a) x(s)+\beta(s-t+a) u(s, s-t+a),
\end{aligned}
$$

and the initial condition becomes $x(0)=y(0, a-t)=\gamma(a-t)$ because of boundary condition (3) given on the segment $\{0\} \times[0, \omega]$. The solution of the motion equation for $t \leq a$ can be obtained by the substitution $x(t)=y(t, a)$, therefore

$y(t, a)=e^{\int_{0}^{t} \alpha(r-t+a) d r}\left\{\int_{0}^{t} \beta(r-t+a) u(r, r-t+a) e^{-\int_{0}^{t} \alpha(v-t+a) d v} d r+\gamma(a-t)\right\}$.

On the other hand, if $t>a$, then the differential equation for the function $x(s)=y(s, s-t+a)$ remains $(5)$, but the initial condition becomes $x(t-a):=$ $y(t-a, 0)=\delta(t-a)$, because we are using the boundary condition (4) given on the semi-axis $(0,+\infty) \times\{0\}$. After some algebraic manipulations, the solution of the motion equation for $t>a$ becomes

$$
y(t, a)=e^{\int_{0}^{a} \alpha(r) d r}\left\{\int_{0}^{a} \beta(r) u(r+t-a, r) e^{-\int_{0}^{s} \alpha(v) d v} d r+\delta(t-a)\right\} .
$$

So that, for any feasible control the explicit solution of the motion equation can be represented using the variation of constants formula, more precisely (6) for $t \leq a$, and (7) for $t>a$ respectively. We notice that: if $\gamma(a) \in$ $C^{0}([0, \omega], \mathbb{R})$ and $\delta(t) \in C^{0}((0,+\infty), \mathbb{R})$ then the solution of $(2),(3)$, and (4) is a differentiable function except in the points of the line $a=t$ where the function is discontinuous when $\gamma(0) \neq \lim _{t \mapsto 0^{+}} \delta(t)$. 


\section{Sufficient conditions}

In order to characterize an optimal solution of the age-structured optimal control problem (1), (2), (3), and (4), we want to use the sufficient conditions described in [3].

Theorem 3.1. Let us assume that we can find a solution $\left(y^{*}(t, a), q^{*}(t, a)\right)$ for the following linear system of PDEs

$$
\left\{\begin{array}{l}
\partial_{t} y(t, a)+\partial_{a} y(t, a)=\alpha(a) y(t, a)-q(t, a) \beta^{2}(a) / \zeta(a) \\
\partial_{t} q(t, a)+\partial_{a} q(t, a)=-\varepsilon(a) y(t, a)+(\rho-\alpha(a)) q(t, a) \\
y(0, a)=\gamma(a), \quad y(t, 0)=\delta(t) \\
q(t, \omega)=0, \quad|q(t, a)|<M \quad \forall t \in[0,+\infty), \forall a \in[0, \omega]
\end{array}\right.
$$

If $\varepsilon(a) \leq 0$ and $\zeta(a)<0$ for all $a \in[0, \omega]$, then the function

$$
u^{*}(t, a)=-\frac{q^{*}(t, a) \beta(a)}{\zeta(a)}
$$

is an optimal control of the problem with motion equation (2), boundary conditions (3), (4), and objective functional (1).

Proof We want to apply Theorem 3.2 proved in [3]. Using the same notation, let us introduce the current value Hamiltonian

$$
H_{c}(a, u, y, q)=\frac{\varepsilon(a)}{2} y^{2}+\frac{\zeta(a)}{2} u^{2}+q(\alpha(a) y+\beta(a) u) .
$$

We notice that the function

$$
u_{c}^{\#}[a, y, q]=-\frac{q \beta(a)}{\zeta(a)}
$$

is well-defined and it is a maximum for the current value Hamiltonian w.r.t. the variable $u$ because $\zeta(a)<0$ for all $a \in[0, \omega]$. With abuse of notation, writing $q^{*}$ instead of $q^{*}(t, a)$, function

$$
H_{c}\left(a, u_{c}^{\#}\left[a, y, q^{*}\right], y, q^{*}\right)=\frac{\varepsilon(a)}{2} y^{2}+\frac{\zeta(a)}{2} \frac{\left(q^{*} \beta(a)\right)^{2}}{\zeta^{2}(a)}+q^{*}\left(\alpha(a) y-\beta(a) \frac{q \beta(a)}{\zeta(a)}\right)
$$

is concave w.r.t. the variable $y$ because $\varepsilon(a) \leq 0$ for all $a \in[0, \omega]$.

We observe that (8) is a linear system of PDEs. We can decouple this system using the standard technique associated to the Riccati equation. 
Theorem 3.2. Let us assume that we can find a solution $\left(y^{*}(t, a), \varphi^{*}(t, a)\right)$ for the system of PDEs

$$
\left\{\begin{array}{l}
\partial_{t} y(t, a)+\partial_{a} y(t, a)=\left(\alpha(a)-\varphi(t, a) \beta^{2}(a) / \zeta(a)\right) y(t, a) \\
\partial_{t} \varphi(t, a)+\partial_{a} \varphi(t, a)=\varphi^{2}(t, a) \beta^{2}(a) / \zeta(a)-(2 \alpha(a)-\rho) \varphi(t, a)-\varepsilon(a) \\
y(0, a)=\gamma(a), \quad y(t, 0)=\delta(t) \\
\varphi(t, \omega)=0, \quad|\varphi(t, a) y(t, a)|<M \quad \forall t \in[0,+\infty), \forall a \in[0, \omega]
\end{array}\right.
$$

If $\varepsilon(a) \leq 0$ and $\zeta(a)<0$ for all $a \in[0, \omega]$, then the function

$$
u^{*}(t, a)=-\frac{\varphi^{*}(t, a) y^{*}(t, a) \beta(a)}{\zeta(a)}
$$

is an optimal control for the problem with motion equation (2), with boundary conditions (3), (4), and with objective functional (1).

Proof With abuse of notation, we write $y^{*}, q^{*}, \varphi^{*}$ instead of $y^{*}(t, a), q^{*}(t, a)$, $\varphi^{*}(t, a)$. Let us assume that $\left(y^{*}, \varphi^{*}\right)$ is a solution of $(10)$, in order to prove the thesis, we have to show that $\left(y^{*}, q^{*}\right)$, with $q^{*}=\varphi^{*} y^{*}$, is a solution of (8). We notice that the first equation in the system (10) is equivalent to the first equation in the system (8). From the definition of $q^{*}$, the following expression on the derivatives holds

$$
\partial_{t} q^{*}+\partial_{a} q^{*}=\left(\partial_{t} \varphi^{*}+\partial_{a} \varphi^{*}\right) y^{*}+\varphi^{*}\left(\partial_{t} y^{*}+\partial_{a} y^{*}\right)
$$

Using (10), we obtain that $\partial_{t} q^{*}+\partial_{a} q^{*}$ is equal to

$$
\left(\left(\varphi^{*}\right)^{2} \beta^{2}(a) / \zeta(a)-(2 \alpha(a)-\rho) \varphi^{*}-\varepsilon(a)\right) y^{*}+\varphi^{*}\left(\left(\alpha(a)-\varphi^{*} \beta^{2}(a) / \zeta(a)\right) y^{*}\right),
$$

rearranging the terms, we get

$$
\partial_{t} q^{*}+\partial_{a} q^{*}=(\rho-\alpha(a)) q^{*}-\varepsilon(a) y^{*},
$$

and the latter is the second equation in the system (8).

Let us observe that the two PDEs in the system (10) are decoupled, hence we can solve the second PDE for the function $\varphi(t, a)$, and then substitute its solution in the first PDE to compute $y(t, a)$. This approach turns out to be useful to find a stationary solution for the optimal control. If we want to consider an equilibrium point for an optimal control, then all the data of the problem have to be autonomous, hence in the following, we assume that $\delta(t)=\delta^{*}$ for all $t \in[0,+\infty)$.

Corollary 3.3. Let us assume that we can find a solution $\left(Y^{*}(a), \Phi^{*}(a)\right)$ for the system of decoupled ODEs

$$
\left\{\begin{array}{l}
Y^{\prime}(a)=\left(\alpha(a)-\Phi(a) \beta^{2}(a) / \zeta(a)\right) Y(a) \\
\Phi^{\prime}(a)=\Phi(a)^{2} \beta^{2}(a) / \zeta(a)-(2 \alpha(a)-\rho) \Phi(a)-\varepsilon(a) \\
Y(0)=\delta(t) \equiv \delta^{*} \\
\Phi(\omega)=0, \quad|\Phi(a) Y(a)|<M \quad \forall a \in[0, \omega]
\end{array}\right.
$$


If $\varepsilon(a) \leq 0$ and $\zeta(a)<0$ for all $a \in[0, \omega]$, and if $\delta(t)=\delta^{*}$ for all $t \in[0,+\infty)$, then the function

$$
u^{*}(t, a)=-\frac{\Phi^{*}(a) Y^{*}(a) \beta(a)}{\zeta(a)}
$$

is an optimal control for the problem with motion equation (2), with boundary conditions $y(0, a)=Y^{*}(a), y(t, 0)=\delta^{*}$ for all $t \in[0,+\infty)$, and with objective functional (1).

Proof If we are looking for an equilibrium point we have to find a solution such that $y(t, a)=Y(a)$ and $\varphi(t, a)=\Phi(a)$. A direct application of Theorem 3.2 allows us to conclude.

We close this section with a simple example in which a closed form solution can be obtained. Let us assume that we want maximize the objective functional

$$
J(u)=\int_{0}^{+\infty} \int_{0}^{\omega} e^{-t}\left[-\frac{1}{2} y^{2}(t, a)-\frac{1}{2} u^{2}(t, a)\right] d a d t .
$$

subject to

$$
\left\{\begin{array}{l}
\partial_{t} y(t, a)+\partial_{a} y(t, a)=y(t, a) / 2-u(t, a) \\
y(0, a)=1 \\
y(t, 0)=1
\end{array}\right.
$$

This problem is an instance of $(1)-(4)$ where $\alpha(a)=1 / 2, \beta(a)=-1, \varepsilon(a)=$ $-1, \zeta(a)=-1, \rho=1$. Applying Corollary 3.3, system (12) becomes

$$
\left\{\begin{array}{l}
Y^{\prime}(a)=(\Phi(a)-1 / 2) Y(a) \\
\Phi^{\prime}(a)=-\Phi(a)^{2}+1 \\
Y(0)=1 \\
\Phi(\omega)=0, \quad|\Phi(a)|<M \quad \forall a \in[0, \omega]
\end{array}\right.
$$

Solving (15), we obtain $\Phi(a)=\tanh (a-\omega)$, and $Y(a)=\cosh (a-\omega) / \cosh (\omega)$. We emphasize that even if the coefficients do not depend on the age, the problem and its optimal path are age dependent.

\section{Conclusions}

In this paper we have shown how to apply the sufficient conditions introduced in [3] to a linear-quadratic age-structured differential games. Using the standard theory of Riccati equation we obtain that the solution of the problem is reduced to the solution of a decoupled system pf PDEs. When the model is autonomous, we can use the results presented in this paper to find the optimal equilibrium. 
The theory described in this paper is the starting point to analyze linearquadratic, age-structured differential games. Some results about this issue can be found in [9], however, to the best of our knowledge, a comprehensive approach to linear quadratic, age-structured differential games is still missing.

\section{References}

[1] Almeder, C., Caulkins, J.P., Feichtinger, and G., Tragler, G., An agestructured single-state drug initiation model-cycles of drug epidemics and optimal prevention programs, Socio-Economic Planning Sciences, 38 (1) (2004), 91-109. https://doi.org/10.1016/S0038-0121\%2803\%29000302

[2] Boucekkine, R., Hritonenko, N., Yatsenko Y., Optimal Control of Agestructured Populations in Economy, Demography, and the Environment, Routledge, 2011.

[3] Buratto A., Grosset, L., and Sartori, E., Current-Value Hamiltonian for an Age_structured Optimal Control Problem, Applied Mathematical Sciences, 14 (3) (2020), 133-140. https://doi.org/10.12988/ams.2020.912171

[4] Engwerda, J., LQ Dynamic Optimization and Differential Games, John Wiley \& Sons, 2005. https://www.wiley.com/enus/LQ+Dynamic+Optimization+and+Differential+Games-p9780470015513

[5] Feichtinger, G., Tragler, G., and Veliov, V.M., Optimality conditions for age-structured control systems, Journal of Mathematical Analysis and Applications, 288 (1) (2003), 47-68.

https://doi.org/10.1016/j.jmaa.2003.07.001

[6] Feichtinger, G., Hartl, R.F. , Kort, P.M. , and Veliov, V., Anticipation effects of technological progress on capital accumulation: a vintage capital approach, Journal of Economic Theory, 126(1) (2006), 143-164.

https://doi.org/10.1016/j.jet.2004.10.001

[7] Grass, D., Caulkins, J.P., Feichtinger, G., Tragler, G., and Behrens, D.A., Optimal Control of Nonlinear Processes, Springer-Verlag, 2008.

https://doi.org/10.1007/978-3-540-77647-5

[8] Grosset, L. and Viscolani, B., Advertising for the introduction of an age-sensitive product, Optimal Control Applications and Methods, 26 (3) (2005), 157-167. https://doi.org/10.1002/oca.758 
[9] Grosset, L. and Viscolani, B., Age-structured linear-state differential games, European Journal of Operational Research, 254 (1) (2016), 269278. https://doi.org/10.1016/j.ejor.2016.03.025

[10] Iannelli M. and Pugliese A., An introduction to Mathematical Population Dynamics, Springer, 2014. https://doi.org/10.1007/978-3-319-03026-5

[11] Krastev, V., Mangasarian-type sufficient optimality conditions for agestructured control problem with state constraints. An application to investment in vintage capital, Serdica Mathematical Journal, 39 (2) (2013), 155-188. http://eudml.org/doc/294971

[12] Krastev, V., Arrow-type sufficient conditions for optimality of agestructured control problem, Central European Journal of Mathematics, 11 (6) (2013), 1094-1111. https://doi.org/10.2478/s11533-013-0219-7

[13] Salsa, S., Partial Differential Equations in Action, Springer, 2008. https://doi.org/10.1007/978-3-319-15093-2

[14] Simon, C., Skritek, B., and Veliov, V., Optimal immigration age-patterns in populations of fixed size, Journal of Mathematical Analysis and Applications, 405 (1) (2013), 71-89.

https://doi.org/10.1016/j.jmaa.2013.03.061

[15] Skritek, B. and Veliov, V.M., On the Infinite horizon Optimal Control of Age-Structured Systems, Journal of Optimization Theory and Applications, 167 (1) (2015), 243-271. https://doi.org/10.1007/s10957-014-0680$\mathrm{x}$

Received: May 27, 2020; Published: June 18, 2020 